\title{
LA INNOVACIÓN EDUCATIVA EN LA ENSEÑANZA SUPERIOR: FACEBOOK COMO HERRAMIENTA DOCENTE.
}

Hipólito Vivar- Zurita1: Universidad Complutense de Madrid. España hvivar@ccinf.ucm.es

Alberto García-García: Universidad Complutense de Madrid. España algarci@ccinf.ucm.es

Natalia Abuín- Vences: Universidad Complutense de Madrid. España nabuinve@ccinf.ucm.es

Raquel Vinader -Segura: Universidad Complutense de Madrid. España rvinader@ccinf.ucm.es

Patricia Núñez-Gómez: Universidad Complutense de Madrid. España pnunezgo@ccinf.ucm.es

Ma Ángeles Martín-Pérez. Universidad Complutense de Madrid. España mariaangelesmartinperez@pdi.ucm.es

\section{RESUMEN}

El Grupo FONTA (Formación en Nuevas Tecnologías del Audiovisual) realiza desde hace varios años investigaciones relacionadas en el campo de la innovación en la educación. Uno de los objetivos del grupo es acercar a la universidad las herramientas que en su vida cotidiana utilizan los alumnos como las redes sociales. En este artículo se describe como ha influido Facebook en el desarrollo de las clases y sus consecuencias para el desarrollo de las mismas.

PALABRAS CLAVE: Innovación Educativa - Redes sociales - Facebook. 


\title{
INNOVATION IN HIGHER EDUCATION: FACEBOOK AS A TEACHING TOOL
}

\begin{abstract}
FONTA Group ( Training in New Audiovisual Technologies ) for several years have been researching in the field of innovation in education. In the last years, those researchs have tried to bring tools that were used in the daily lives of students such as social networks. This article describes how Facebook has influenced in the development and implications of classes.
\end{abstract}

KEY WORDS: Innovation in Education - Social Networks - Facebook

\section{INTRODUCCIÓN}

Las nuevas tecnologías están provocando profundos cambios en las relaciones entre usuarios: las redes sociales, que convierten al usuario en gestor de contenidos, y el uso de entornos virtuales para desarrollar las interacciones personales, están revolucionando el panorama comunicativo a escala global. De manera específica, la formación y la educación en este terreno adquieren una relevancia especial porque se están definiendo nuevos modelos de negocio que irán creando un nuevo panorama de interrelaciones culturales y económicas entre los diferentes nacionalidades.

Además de incorporar esta formación a los planes de estudio, una de las estrategias a seguir a la hora de formar profesionales expertos en los nuevos medios, es utilizar estas tecnologías en el momento de la formación. Los videojuegos, los mundos virtuales y las redes sociales son herramientas que pueden ayudar a incrementar el rendimiento de los alumnos y favorecer no sólo la interacción con el profesor sino entre los propios estudiantes, algo que mejora el intercambio de conocimientos entre los mismos y ayuda a mejorar los resultados finales en las diferentes asignaturas. Por otra parte el uso de estas herramientas favorece la incorporación al mercado laboral de profesionales que dominan las nuevas tecnologías de la información lo que les facilitará la inserción laboral en un mercado dominado por los nuevos medios. 
En este sentido, se plantean no sólo nuevas necesidades sino nuevas posibilidades para optimizar la formación y su aplicación directa a los nuevos modelos de negocio emergentes. Un ejemplo significativo son las relaciones tecnológicas que están implícitas en las redes sociales y que conllevan un sustancial cambio en las formas de participación, haciendo de las mismas un instrumento educativo y de trabajo que, de manera polivalente, ayudan al usuario a integrarse en todos los aspectos personales y profesionales.

Convertir a los internautas en gestores de contenidos de manera que puedan modificar «todas aquellas utilidades y servicios de Internet que se sustentan en una base de datos, ya sea en su contenido (añadiendo, cambiando o borrando información o asociando metadatos a la información existente), bien en la forma de presentarlos, o en contenido y forma simultáneamente» (Ribes, 2007), implicó pasar de un mundo de poder descentralizado a otro de poder distribuido (Ugarte, 2007).

Estas nuevas estructuras son socialmente más potentes e innovadoras para el trabajo en red (Santamaría, 2008) y son propicias para la creación de comunidades de aprendizaje y redes de conocimiento.

En este estudio analizamos la implantación de las redes sociales en la docencia universitaria como herramienta de aprendizaje. Para conocer la percepción y el uso que los estudiantes hacen de esta herramienta se virtualizaron varias asignaturas de las titulaciones de Publicidad y Comunicación Audiovisual durante los cursos académicos 2009/2010 y 2010/2011.

Para obtener los resultados se llevaron a cabo más de quinientas encuestas antes y después de la utilización de Facebook. Los resultados apuntan a que tras la reticencia inicial, el uso de esta red social es altamente eficaz para compartir conocimiento e incrementar la motivación de los estudiantes universitarios, produciéndose sinergias efectivas en la creación de contenidos digitales de los estudiantes en relación con el ámbito de estudio de cada asignatura. De esta forma, se desarrollan sistemas de creatividad capaces de estimular nuevos roles de actuación entre los alumnos.

En este año académico y por segunda vez se implementó la red social Facebook dentro de las clases de diferentes asignaturas para alcanzar los siguientes objetivos:

a) Dotar de herramientas integradoras a los alumnos para mejorar en las aulas y en los procesos de estudio de acuerdo con el Espacio Europeo de Educación Superior. Eso supone una mavor motivación para el alumno v facilidad para 
b) Investigar el uso de las redes sociales en educación y proponer un modelo de mejora. La experiencia del grupo en la implantación de las Nuevas Tecnologías hace que el método vaya mejorando año tras año por el feedback de los alumnos y el contacto de los diferentes miembros del proyecto con grupos de investigación nacionales e internacionales que realizan el mismo estudio en sus universidades.

c) Explorar la posibilidad de interactuación de diferentes soportes conectados con redes sociales, tales como el móvil. En la actualidad, según con los objetivos propuestos, se han desarrollado concretamente algunos encuentros en plataformas virtuales y un taller de geolocalización para probar la interacción entre las redes sociales y otro tipo de soportes.

d) Formar a alumnos en el uso de las redes sociales como herramienta de comunicación en la empresa. En este sentido es necesario resaltar que uno de los perfiles profesionales emergentes y con un elevado índice de demanda en el mercado laboral es el de Community Manager.

\section{METODOLOGÍA}

Como refuerzo a la docencia tradicional, los profesores de las asignaturas Teoría de la Publicidad, Programación de Radio y TV, y Tecnologías de los Medios Audiovisuales utilizaron la red social Facebook. Estas tres asignaturas son impartidas por el Departamento de Comunicación Audiovisual y Publicidad 1 (CAVP 1). A fin de evaluar la opinión de los alumnos sobre la iniciativa, se les pidió que completasen un cuestionario al inicio del curso, y posteriormente se repartió el mismo cuestionario al final del mismo. Como ya se ha dicho anteriormente, el proyecto que se presenta tiene como objetivo mejorar la interacción entre docente y alumno con el fin de alcanzar las competencias designadas por los profesores por áreas de conocimiento y de cara a la implantación del Espacio Europeo de Educación Superior (EEES).

La página de las asignaturas se confeccionó como un microsite. El término microsite se refiere a un diseño Web determinado, que consiste en un espacio individual o conjunto de páginas que funcionan como suplemento auxiliar de una Web primaria. Aunque, por lo general, la página principal tiene su propio nombre de dominio o subdominio, los microsites suelen emplearse con fines comerciales o de marketing.

La utilización de la red social Facebook, nos permite por sus características tener la página dinamizada y nos facilita la conexión con el grupo así como comunicar información sobre la asignatura, eventos y demás información docente. Los alumnos, al ser una herramienta habitual de comunicación pueden utilizarla de manera 
La investigación consta de un pre- test para conocer la opinión que los alumnos tienen a priori sobre esta herramienta educativa. En una segunda fase se pasa a dinamizar la asignatura por parte del docente y más tarde con la colaboración del alumnado. Por último se les pasa un cuestionario, un post-test donde evalúan su experiencia. Pasamos a describir a continuación todo el proceso:

Etapa 1.- Diseño de pre-test. El cuestionario (tanto pre-test como post-test) se compone de 5 preguntas de respuesta cerrada o semicerrada, más una $6^{a}$ cuestión que puede responderse de forma abierta. De las primeras 5 preguntas, 3 de ellas tenían las opción de añadir una breve justificación de la respuesta.

Asimismo, 4 de estas 5 preguntas eran de respuesta única, y sólo una permitía respuesta múltiple. Además, los alumnos debían indicar a qué asignatura pertenecían. De forma automática se añadía la fecha y hora de respuesta del cuestionario.

Se les pasó este test a los alumnos cuyos profesores estaban implicados en el proyecto, se colgó en las páginas de las diferentes asignaturas y los alumnos lo rellenaban antes de dinamizar la página. Se pretende conocer su opinión sobre el uso de esta herramienta en la docencia y compararla con el campus virtual. Se miden los conocimientos previos y expectativas sobre su uso, así podemos comparar los resultados después de usarla.

Etapa 2.- Dinamización de asignaturas. Durante todo el cuatrimestre los docentes procedieron a la dinamización del espacio virtual colgando textos y material audiovisual utilizado en el aula. Los propios alumnos realizan el trabajo de Community Manager introduciendo enlaces, videos y demás material que supervisa el profesor.

Etapa 3.- Diseño de post-test. Posteriormente, se les pasa un post-test para evaluar la experiencia, comprobar diferentes ítems, tales como motivación, expectativas y utilidad de la herramienta. En este caso, se colgó el test también en las páginas de Facebook y los alumnos podían completarlo.

Etapa 4.- Recogida de datos y evaluación de resultados. En el caso de las dos pruebas, la red social Facebook, a través de Facebook Insights, permite recoger los resultados y realizar estadísticas sobre el número de alumnos que han rellenado el test, participación, segmentación por sexo y edad, además de estadísticas sobre las preguntas realizadas. Todo se recoge en diferentes gráficos que permiten al investigador observar los resultados de manera más eficaz. Dentro de cada página de Facehonk se enc11entra la onción de visıalizar el norcentaie de la actividad realizada 
Así, hemos podido saber quién ha introducido "Me gusta" y cuántos, una vez seleccionado, han decidido retirarlo. Sabremos, de paso, el sexo y la edad de los usuarios y las pestañas en las que más pinchan. Este último dato nos permitiría cambiar toda nuestra estrategia si, de repente, descubrimos que hay una parte de nuestra página por la que nadie se interesa.

El cuestionario fue respondido de forma voluntaria por los alumnos. En la $1^{\mathrm{a}}$ ola (principio de curso) hubo un total de 228 respuestas. Por su parte, en la $2^{\mathrm{a}}$ ola (final de curso) los alumnos que respondieron fueron 149. Esta variación se ha tenido en cuenta a la hora de interpretar los porcentajes.

\section{ANÁLISIS Y DISCUSIÓN}

A continuación enumeramos los principales resultados relacionados con las preguntas del cuestionario.

a) ¿Crees que puede tener ventajas el empleo de Facebook como apoyo a una clase tradicional?

Una amplia mayoría de alumnos se muestra favorable a las ventajas de Facebook como apoyo a las clases tradicionales. Así, en la $1^{\text {a }}$ ola, hubo 217 respuestas afirmativas $(95 \%)$ frente a sólo 11 respuestas negativas. Una vez realizada la experiencia, el número de respuestas negativas subió a 24 (16\%), aunque sigue siendo un porcentaje minoritario.

b) ¿Crees que puede tener desventajas el empleo de Facebook como apoyo a una clase tradicional?

Esta segunda pregunta iba dirigida a localizar desventajas concretas percibidas por el alumnado de cara a usar Facebook como complemento de las asignaturas. En ambas olas (antes y después de la experiencia) el porcentaje de alumnos que encuentra desventajas en el uso de Facebook es muy similar (26 y 25\% respectivamente). En cambio, las observaciones de los alumnos han variado sensiblemente entre la $1^{\mathrm{a}}$ y la $2^{\mathrm{a}}$ ola (antes y después de la experiencia).

En los momentos previos al uso de Facebook como complemento a las asignaturas, los alumnos se mostraban escépticos en diferentes temas. La mayoría de las opiniones se relacionaban con una desventaja que la hemos nombrado "distracción", la distracción que supone Facebook para estudiar una asignatura, ya que esta red social es percibida por los estudiantes como una herramienta de ocio que interfiere con sus deberes académicos (estudio tradicional). 
Esta distracción incluye el "trabajo añadido" que les supondría: utilizar una herramienta más, realizar rutinas diferentes o añadidas a sus rutinas de estudio, etcétera. La segunda desventaja más mencionada por los alumnos es la "universalidad" (o falta de ella) de la red Facebook. Alegaban que no todos los alumnos tenían cuenta en Facebook. Muy relacionada con esta "universalidad" está la tercera desventaja por orden de importancia, el conocimiento de la herramienta (posiblemente, con respecto a la red social Tuenti, altamente popular en el rango de edad entrevistado). De hecho, si se combinan estos dos factores, "no universalidad" y conocimiento de uso, se convierten en la primera objeción.

Otro tema destacado es la privacidad de la red, tanto en términos generales (la privacidad de los datos en Facebook es más laxa que en su competidora Tuenti, que acata la ley española en este tema) como de cara a permitir que los profesores puedan tener acceso a sus datos personales.

Detrás de estos temas, las desventajas son menos significativas, aunque pueden mencionarse la redundancia de la herramienta al existir un campus virtual, las limitaciones que pueda tener Facebook o la posibilidad de que la herramienta se convierta en un "sustituto" de las clases y desmotive la asistencia.

En la segunda ola, en cambio, la mayoría de las objeciones no han sido para la herramienta en sí, sino para el uso que se ha hecho de ella. La mayor parte de las quejas sobre el uso han ido dirigidas a que los profesores no cumplieron el compromiso de puntualidad (compromiso que ellos sí les exigen a los alumnos) y a falta de feedback de la herramienta, al no poder publicar en el muro ni obtener respuesta de profesores por ese canal. No obstante, se han repetido algunas observaciones como la distracción y el trabajo añadido que supone, y la redundancia con el Campus Virtual. Hay que tener en cuenta que el número de respuestas en la segunda ola es mucho menor de cara a evaluar las respuestas abiertas.

\section{c) ¿Qué crees que puede ser más útil para la docencia?}

Se preguntó a los alumnos cuál de las dos herramientas de la red usadas en ese momento (la red Facebook o el Campus Virtual de la Universidad Complutense) encontraban más provechosas para complementar las clases. Las posibles respuestas eran Facebook, Campus Virtual, Ambas o Ninguna. Mientras que en la $1^{\mathrm{a}}$ ola la mayoría de los alumnos prefería ambas herramientas (73\% con 166 votos), este porcentaje ha retrocedido notablemente en la $2^{\circ}$ ola (56\% con 84 votos). La preferencia por el Campus Virtual ha ganado en porcentaje aunque no en número de votos, mientras que Facebook ha contado con más respuestas favorables en la $2^{\mathrm{a}}$ ola. Parte de este cambio puede explicarse mediante las respuestas abiertas de los encuestados. 
Ya antes de haber utilizado la experiencia, los alumnos manifestaron que si bien el Campus Virtual era una herramienta a priori más idónea para complementar la asignatura, en la práctica tenía muchos problemas de funcionamiento (lentitud, conectividad) y no era usado de forma habitual por los profesores. Estas observaciones se repitieron en menor medida una vez realizada la experiencia. Aunque no era lo que se preguntaba, hubo alumnos que sugirieron el uso de otras herramientas. Las que se han propuesto más veces han sido Tuenti, blogs o páginas web personales de los profesores, y Twitter.

\section{d) ¿Cuáles de estas metodologías docentes crees que son más eficaces?}

Se proponían una serie de herramientas y metodologías para que los alumnos escogiesen las que a su juicio serían más eficaces. La principal preferencia es el apoyo de las clases con material audiovisual, seguido del apoyo de las clases con redes sociales o bien con el apoyo del Campus Virtual.

Al haber un menor número de respuestas en la segunda ola, todas las opciones han reducido el número de respuestas, y al tratarse de una pregunta con opción de respuesta múltiple, eso ha supuesto un descenso en el porcentaje de todas las preferencias con respecto a la $1^{\mathrm{a}}$ ola, a excepción de la respuesta "Lección tradicional sin apoyo audiovisual", que a pesar de todo refleja un porcentaje casi marginal de preferencia.

Tabla 1. Respuestas

\begin{tabular}{|c|c|c|c|c|}
\hline & \multicolumn{2}{|l|}{$1^{\mathrm{a}}$ ola } & \multicolumn{2}{|l|}{$2^{\mathrm{a}}$ ola } \\
\hline & $N^{o}$ de respuestas & Porcentaje & $N^{o}$ de respuestas & Porcentaje \\
\hline $\begin{array}{l}\text { Lección tradicional con } \\
\text { apoyo audiovisual }\end{array}$ & 195 & $86 \%$ & 115 & $77 \%$ \\
\hline $\begin{array}{l}\text { Lección tradicional sin } \\
\text { apoyo audiovisual }\end{array}$ & 2 & $1 \%$ & 6 & $4 \%$ \\
\hline Talleres & 132 & $58 \%$ & 41 & $28 \%$ \\
\hline Seminarios & 72 & $32 \%$ & 15 & $10 \%$ \\
\hline $\begin{array}{l}\text { Con apoyo de redes } \\
\text { sociales }\end{array}$ & 147 & $64 \%$ & 95 & $64 \%$ \\
\hline $\begin{array}{l}\text { Con apoyo del Campus } \\
\text { Virtual }\end{array}$ & 138 & $61 \%$ & 76 & $51 \%$ \\
\hline
\end{tabular}

e) ¿Crees que Facebook puede ser una buena herramienta docente? 
Con todo, el porcentaje de respuestas afirmativas es claramente superior en ambos casos.

Los alumnos tenían la opción de explicar su respuesta, ya fuese afirmativa o negativa. Ha sido una de las preguntas con mayor número de respuestas abiertas, por encima incluso de la pregunta 6. Resulta muy interesante comprobar la evolución de las opiniones de los alumnos entre la primera y la segunda ola, tanto en los casos favorables como en los desfavorables .

Los alumnos que opinaban que Facebook podría ser una buena herramienta docente (primera ola, antes de realizarse la experiencia) basaban su respuesta fundamentalmente en los beneficios que aportan las nuevas tecnologías de la información y la comunicación y las redes sociales. Uno de los aspectos más valorados era la posibilidad de interacción, ya fuera con los profesores (feedback) o entre los propios alumnos. En definitiva, se valoraba la posibilidad de tener un canal de comunicación añadido.

Otros beneficios que Facebook aportaba como red social eran la "flexibilidad", entendida no sólo como flexibilidad en el horario (puede ser consultada a cualquier hora del día) sino como las posibilidades añadidas de interacción y contenidos.

También se valoró la rapidez del espacio 2.0 (instantáneo) frente a otros métodos convencionales, como dejar apuntes en reprografía. La posibilidad de publicar material complementario también fue muy valorada. Uno de los beneficios más valorados, en términos cuantitativos, fuera de las ventajas genéricas que aporta el uso de una red social, era que la plataforma tiene un uso muy extendido (alto porcentaje de penetración entre los usuarios de internet) y es fácil de utilizar.

Curiosamente, esto contrasta con las reticencias mostradas en las respuestas a la pregunta $n^{\circ} 2$, que señalaban que la plataforma Facebook no era usada por todos los alumnos. De hecho, como argumento en contra se ha mencionado la necesidad de crear una cuenta a propósito para poder seguir la asignatura. Otro argumento que se interpretó tanto a favor como en contra fue que Facebook es una plataforma en la que el alumno pasa mucho tiempo.

Esto podía verse como algo positivo, ya que era una forma de estar siempre al tanto de las novedades que había en la asignatura (e incluso llega a señalarse que al mezclarse con el ocio, predispone y motiva más al alumno para el estudio). De la misma forma, algunos alumnos opinaban que en Facebook, debido a la gran 
No obstante, se valoró positivamente (siempre hablando de la primera ola) la forma en que Facebook podría agilizar el seguimiento y el estudio de las asignaturas, así como la posibilidad de conseguir una mayor participación por parte del alumnado.

Por último, un número importante de respuestas aludían a las ventajas de Facebook frente al Campus Virtual. La mayoría de este tipo de respuestas se debían a una mayor fiabilidad y rapidez de la red social frente a la plataforma de la UCM, pero también se mencionó una mayor familiaridad y facilidad de uso de Facebook, e incluso un diseño más atractivo e intuitivo.

En la segunda ola, los argumentos a favor se mantenían en unas proporciones similares. La interactividad (profesores-alumnos y alumnos-alumnos) siguió siendo el aspecto mejor valorado, esta vez con una clara ventaja con respecto a la facilidad de uso de Facebook y su alto grado de penetración. Estos dos argumentos (interactividad por un lado, y uso fácil y muy extendido por el otro) tenían prácticamente el mismo protagonismo en la primera ola.

El hecho de que Facebook ayudaba el seguimiento y el estudio de la asignatura continuó siendo muy valorado (teniendo en cuenta el número de respuestas totales en la segunda ola, este argumento ganó en porcentaje). En relación a esto se mencionó la facultad de la red social como motivador para seguir la asignatura y participar. Por su parte, la posibilidad de publicar material complementario redujo el número de menciones, así como la mayor fiabilidad de Facebook frente al Campus Virtual.

Como argumentos en contra, en la primera ola destacaron la "distracción", que ya se ha citado, que supone Facebook (usar una herramienta que se asocia al ocio y tiempo libre para actividades de estudio) y la necesidad de crear una cuenta en una red social para seguir una asignatura (ya fuese porque el alumno no era usuario de Facebook o porque se optase por crear una segunda cuenta debido a razones de privacidad).

Como se ha señalado, estos argumentos han sido interpretados como elementos positivos por otros alumnos. Cabe señalar que la reticencia a mezclar las actividades académicas con una herramienta percibida para el ocio ya se había manifestado en anteriores preguntas. También hubo alumnos que opinaron que el uso de Facebook como herramientadocente era innecesario ya que existía otra herramienta para tal efecto, el Campus Virtual.

Curiosamente, incluso en esos casos hubo menciones al mal funcionamiento del

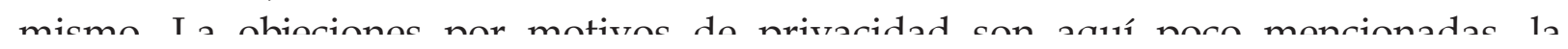


En la segunda ola del cuestionario, las objeciones al uso de Facebook como herramienta docente se centraron más que en las posibilidades de la red social en sí (que algunos aún consideraron insuficiente y poco adecuada para los requisitos de las asignaturas), en el uso que se había hecho de Facebook durante la experiencia. Hubo varias quejas sobre la interacción entre los alumnos (o más bien la falta de interacción) y especialmente hacia la ausencia de feedback por parte de los profesores, una retroalimentación que esperaban tener al usar este canal de comunicación.

Del resto de argumentos, en todos los casos se reduce el número de menciones, hasta el punto que algunos como la privacidad desaparecen de la lista. Las quejas debidas a la "distracción" que supone el uso obligatorio de Facebook para el alumno se reducen a un tercio, mientras que las opiniones acerca de que es una herramienta redundante (con respecto al Campus Virtual) o que supone la obligación de crear una cuenta expresamente para la asignatura se manifiestan la mitad de las veces.

\section{f) ¿Qué aplicaciones añadirías?}

Esta última pregunta, de respuesta abierta, permitía a los alumnos opinar sobre otras aplicaciones que pudieran ser útiles en la docencia. A la hora de responder esta pregunta (téngase en cuenta que no todos los alumnos han contestado la pregunta 6), en la mayoría de los casos los comentarios no aluden a aplicaciones concretas sino a posibles usos de las aplicaciones, sin especificar si se refieren a Facebook, el Campus Virtual $\mathrm{u}$ otras, e incluso a mejoras en el funcionamiento de la propia asignatura. Catalogar las respuestas que aludían a esas mejoras no resulta fácil por la variedad y vaguedad de las respuestas.

De forma orientativa, se pueden señalar algunos posibles usos y mejoras que han propuesto los alumnos. Así, en la primera ola del cuestionario se sugerían la subida a la aplicación (no se especificaba si se trataba de Facebook o de otras aplicaciones) de apuntes, vídeos, enlaces a software y eventos relacionados con las asignaturas (congresos, seminarios) y en definitiva material complementario. También se ha aludido en varias ocasiones al uso de la aplicación para colgar exámenes, preguntas y respuestas de ejercicios, novedades, incidencias, etcétera; básicamente, se buscaba el uso de Facebook como canal para llevar el día a día de la asignatura.

En la segunda ola del cuestionario, dentro de las posibles mejoras del uso de la aplicación se señalaban la publicación de enlaces a software relacionado con las asignaturas e información extra. También volvió a mencionarse que una herramienta como Facebook debería proporcionar un feedback con el profesor. La lista de aplicaciones concretas sugeridas por los alumnos es larga, si bien cada aplicación es nombrada en muy pocas ocasiones. De hecho, el total de respuestas que mencionan 
Así, Tuenti es la aplicación más mencionada (5 respuestas), seguida de Twitter (4 menciones, una de ellas indicando que esa aplicación y Facebook deberían estar viculadas). Con 3 alusiones están Youtube, por un lado; y un Campus Virtual "alternativo" (de nuevo, se está aludiendo a la poca fiabilidad y funcionalidad del actual). También se mencionan las aplicaciones Google Reader; servidores para alojar contenido en red y compartirlo (como podrían ser Rapidshare, Megaupload, etc); uso de blogs; videoconferencias o emisión de clases en streaming; Google Docs; Slide Share; uso de chats; servidores FTP (file transfer protocol); listas de correo; espacios virtuales; foros; iTunes (como servicio para recibir videocast de clases de otras universidades); una "pizarra online" (el alumno sugiere esta aplicación como idea, pero no especifica más detalles); e incluso una respuesta afirma que con el actual Campus Virtual sería suficiente.

En la segunda ola, dado el número menor de respuestas, la lista de aplicaciones también es menor. A la cabeza siguen estando las redes sociales Twitter y Tuenti, aunque esta vez es Twitter la que es mencionada más veces (hay más alusiones que en la primera ola, a pesar del menor número de respuestas totales).

Se vuelven a mencionar el Campus Virtual "alternativo"; Youtube; los servidores para alojar contenido; Google Docs, y el uso del chat. Además, aparecen nuevas aplicaciones como la red Gnoss y FB Question. Se menciona de nuevo que el Campus Virtual es suficiente (otra respuesta indica que basta con tener el $e$-mail de contacto del profesor). El resto de aplicaciones desaparecen.

\section{CONCLUSIONES}

Las siguientes conclusiones se han extraído en base a la interpretación de las respuestas recibidas, no se ha realizado ningún análisis estadístico de los datos. Por lo tanto, estas conclusiones han de tomarse de forma provisional y como posibles líneas generales para un análisis más fundamentado.

A esto hay que añadirle las limitaciones metodológicas de la propia encuesta. En primer lugar, la muestra es autoseleccionada, con el sesgo que eso supone. En segundo, la variación del número de alumnos que respondieron al cuestionario en la $1^{\mathrm{a}}$ y $2^{\mathrm{a}}$ ola, siendo la última sensiblemente menor.

Facebook es percibido como una herramienta de ocio, y además tiene un alto índice de uso entre la población y sus usuarios pasan mucho tiempo en ella. Esto se ha podido comprobar en las respuestas cualitativas de las preguntas 2 y 5 fundamentalmente. Esta creencia generalizada se interpreta como algo positivo pero 
a) Se valora como algo positivo ya que el alumno, al usar la red social a diario, podrá seguir los avances de la asignatura de forma natural. Al integrarse en un espacio fundamentalmente dedicado al ocio, la materia académica se percibe de otra manera y el alumno está mejor predispuesto a estudiar.

b) De la misma forma, otros alumnos consideran que los temas académicos deben estar separados de las herramientas de ocio, y que seguir una asignatura en una red social fomentará las distracciones (alertas, chat, notificaciones, etcétera).

En términos generales, se aprecia una amplia aceptación del uso de Facebook como herramienta docente, tanto en la primera como en la segunda ola. No obstante, el grado de escepticismo crece en la segunda ola si se comparan porcentajes (preguntas 1 y 5). Algunas posibles explicaciones sobre este mayor descontento son:
a) Menor número de respuestas en la segunda ola (y diferente muestra autoleseccionada)
b) La experiencia de usar Facebook como heramienta docente no ha cubierto las expectativas del alumno.
c) Cautela de los alumnos al responder en la primera ola, al quedar varios meses de curso por delante. los alumnos se muestran escépticos ya que no todos son usuarios de Facebook.
d) Parece haber algunas contradicciones en ciertos aspectos. En algunas preguntas,

Sin embargo, en otras se alaba la "universalidad" de esta red social como una de sus ventajas. Aunque a priori, el Campus Virtual es una herramienta más apropiada, los alumnos están descontentos de forma generalizada con su funcionamiento. De ahí que Facebook se prefiera como herramienta docente: es más rápida, ágil, es más usada (incluso por parte de los propios profesores) y en general es más fiable (al tratarse de una red social usada por millones de personas, tiene un equipo de gente en proporción que se encargan de que funcione correctamente las 24 horas del día).

Como norma, el uso de Facebook se valoraba positivamente como la existencia de un canal de comunicación adicional y la posibilidad de interactuar tanto con los profesores como entre los propios alumnos. Muchas de las ventajas adicionales se correspondían con las ventajas genéricas de las redes sociales y las TIC: interactividad, rapidez, etcétera. 
Por el contrario, las quejas sobre el uso de Facebook como herramienta docente derivan de cómo ha sido la experiencia y la forma en que al final se ha utilizado la red social más que de la iniciativa en sí. Entre otras quejas, está la ausencia del feedback que habían esperado los alumnos al disponer de este canal (preguntas 5 y 6), y la falta de puntualidad por parte de los profesores (al usar una herramienta horizontal de comunicación como una red social, los alumnos esperaban una comunicación igualmente horizontal, por lo que también esperaban un compromiso de puntualidad mutuo que han visto incumplido).

Por sugerencias de los alumnos, durante este curso se valorará la implantación de la red social Tuenti puesto que tiene un alto grado de penetración en los jóvenes entre 16 y 24 años. Los alumnos podrían sentirse más cómodos con una herramienta que utilizan más y que se percibe como más segura en aspectos de privacidad.

\section{REFERENCIAS}

Gongadharbatla, H. (2008). Facebook me: collective self-steem,need to belong and internet self-efficacy as predictors of the igeneration's attitudes towards social networking. Journal of Interactive Advertising, 8(2): 5-15.

Mazer,J. P; Murphy,R. E. \& Simonds, C. J. (2007). I'1l see you on facebook: the effects of computer-mediated teacher self-disclosure on student motivation, affective learning and classroom climate. Communication Education, 56(1): 1-17.

Rives, X. (2007). La web 2.0. El valor de los metadatos y de la inteligencia colectiva. Telos, 73.

Santamaria, F (2009). Las redes sociales aplicadas a la práctica docente. Didáctica, Innovación y multimedia, 13.

Ugarte, D. (2007) El poder de las Redes. Madrid: Icaria.

\section{Hipólito Vivar Zurita}

Director del Departamento de Comunicación Audiovisual y Publicidad 1. Catedrático de Tecnología de los Medios Audiovisuales. Profesor de la Asignatura “Tecnología de los Medios Audiovisuales" en el Departamento de Comunicación Audiovisual y Publicidad 1 de la UCM. Director del Grupo de Investigación FONTA (Formación en Nuevas Tecnologías del Audiovisual). 
Comunicación Audiovisual y Publicidad 1 de la UCM. Miembro del Grupo de Investigación FONTA (Formación en Nuevas Tecnologías del Audiovisual).

\section{Natalia Abuín Vences}

Licenciada en Comunicación Audiovisual por la UCM. Doctora en Ciencias de la Información por la UCM. Profesora de "Nuevas Tecnologías de los Medios Audiovisuales" en el Departamento de Comunicación Audiovisual y Publicidad 1 de la UCM (curso 2010-2011) y de "Empresa audiovisual" en el CES Felipe II (UCM). Miembro del grupo de Investigación FONTA (Formación en Nuevas Tecnologías del Audiovisual).

\section{Raquel Vinader Segura}

Licenciada en Comunicación Audiovisual por la UCM. Doctora en Ciencias de la Información por la UCM. Profesora de "Nuevas Tecnologías de los Medios Audiovisuales" en el Departamento de Comunicación Audiovisual y Publicidad 1 de la UCM (curso 2010-2011) y de "Empresa audiovisual" en el CES Felipe II (UCM). Miembro del grupo de Investigación FONTA (Formación en Nuevas Tecnologías del Audiovisual).

\section{Patricia Núñez Gómez}

Licenciada en Publicidad por la UCM.Doctora en Ciencias de la Información por la UCM. Profesora de "Teoría de la Publicidad" y de "Modelos de Análisis Publicitario" en el Departamento de Comunicación Audiovisual y Publicidad 1 de la UCM. Miembro del grupo de Investigación FONTA (Formación en Nuevas Tecnologías del Audiovisual) 